\title{
A WEB-BASED PLATFORM FOR MANAGEMENT AND VISUALIZATION OF GEOMETRIC DOCUMENTATION PRODUCTS OF CULTURAL HERITAGE SITES
}

\author{
C. Ioannidis ${ }^{1}$, I. Tallis ${ }^{2}$, I. Pastos ${ }^{3}$, A.-M. Boutsi ${ }^{1}$, S. Verykokou ${ }^{1}$, S. Soile ${ }^{1}$, P. Tokmakidis ${ }^{2}$, K. Tokmakidis ${ }^{2}$ \\ ${ }^{1}$ Laboratory of Photogrammetry, School of Rural and Surveying Engineering, National Technical University of Athens, Greece - \\ cioannid@survey.ntua.gr, iro_mpoutsi@outlook.com, st.verykokou@gmail.com,ssoile@survey.ntua.gr \\ ${ }^{2}$ Laboratory of Topography, School of Rural and Surveying Engineering, Aristotle University of Thessaloniki, Greece - \\ ioannistallis@gmail.com, ptokmaki@gmail.com, ktok@topo.auth.gr \\ ${ }^{3}$ Department of Cultural Technology and Communication, University of Aegean, Greece - ilias@aegean.gr
}

Commission II, WG II/8

KEY WORDS: Cultural Heritage visualization, web application platform, spatial data, RDBMS, 3D viewer, multi-users

\begin{abstract}
:
The mission of digital Cultural Heritage $(\mathrm{CH})$ repositories goes beyond engagement and dissemination; their research data should be approached and correlated spatially and semantically in the service of conservation and accessibility. Therefore, a flexible system is needed to expand, reuse and repurpose their content, addressing online collaboration, real time progress and ease of use. Towards this direction, the "METEORA" web-based platform is a novel and holistic approach to the organization, management and visualization of the $3 \mathrm{D}$ and $2 \mathrm{D}$ documentation products of $\mathrm{CH}$ sites. It integrates a multi-scale 3D viewer based on the 3DHOP framework, personalized information access and interactive tools for data retrieval and presentation. Emphasis is given in the development of a mid-level interface for creating, updating and maintaining 3DHOP's functionalities and customizing information based on the user's scientific specialty and field of interest. The proposed admin control panel updates the state of the current visualization, allows the integration of 3D models and multimedia and automates the creation of clickable points of interest on top of the 3D surfaces. It is based on a MySQL Relational Database Management System (RDBMS) that supports the back-end User Interface (UI) with create, read, update and delete (CRUD) management functions of a typical RESTful service. Moreover, all the control mechanisms as well as the procedures of authentication, authorization and encryption of the credentials of the certified users are configured through the PHP scripting language and JSON files. The resulting platform achieves maximum adaptability in digital archiving of heterogeneous datasets and has increasing efficiency in representing all types of data supported by 3DHOP.
\end{abstract}

\section{INTRODUCTION}

Technological innovations in Photogrammetry, Computer Vision and Computer Graphics are enabling new understandings of Cultural Heritage $(\mathrm{CH})$. Sophisticated sensors mounted on cameras, Unmanned Aerial Vehicles (UAVs) and laser scanners have made data collection more efficient, cost-effective and accurate. In turn, equally innovative computing and rendering technologies are transforming these datasets into detailed maps, point clouds and 3D meshes, which can be accessed by the public via the web. Both researchers and professionals as well as nonexperts benefit from the high-quality that characterizes every stage. Conservation efforts are improved and enhanced with realtime monitoring and diagnosis, immersive virtual experiences and educational content. The several possibilities of representing $\mathrm{CH}$ are introduced by digital libraries, 3D repositories, virtual museums and serious games (Pujol \& Lorente, 2014; Statham, 2019; Champion \& Rahaman, 2020). These platforms must contend with storing, organizing and making accessible different types of heritage data, adopting effective content management strategies. However, their management systems have either limited functionalities due to the restrictions of relational databases and the nature of integrated information or lie exclusively on front-end technologies.

The METEORA project aims at the creation of a web-based platform for the management, visualization and dissemination of the products of the multi-level documentation of the UNESCO site of Meteora. The photogrammetric 3D models of the study area, combined with historical, religious, cultural and geopolitical information, establish the knowledge base and the context of interaction and engagement. The 3D visualization along with the various services for data retrieval, virtual navigation and immersion are implemented with the use of the 3D Heritage Online Platform (3DHOP). The platform is completed by its back-end interface and administration panel for organizing, authoring and updating structured data in a welldefined SQL-based database.

Unlike similar endeavors towards $\mathrm{CH}$ publication, it offers a level of personalization and automation in authoring, managing and uploading 3D and 2D content. This paper focuses on the development of a mid-level software that abstracts database functions, maintains a multi-user front-end interface and represents all types of data supported by 3DHOP. This web management environment of 3DHOP creates and devises all the correlations between the $3 \mathrm{D}$ scene, the points and the spatial entities in real time. Its interactive user interface permits the dynamic preview of 3D models, hotspots and relative multimedia in various viewports before publishing. Thus, the CRUD controls, the PHP and AJAX (Asynchronous JavaScript \& XML) methods for data management as well as the insertion, modification and querying of the multi-modal content are automated within a higher-level infrastructure.

The contributions of this work can be summarized as follows:

i. The proposed back-end system allows dynamic content management, automation of 3DHOP's operations regarding 
3D data uploading and hotspots defining, real-time preview of the 3D scene as well as extensibility at all levels (i.e., new data types, query languages, storage and access methods).

ii. An innovative data loss control mechanism that is enabled while running multiple application's instances in different browser tabs has been developed.

iii. The content and interactive services of the platform are differentiated, based on the scientific specialty and the field of interest of the users.

\section{RELATED WORK}

In recent years, several web-based $\mathrm{CH}$ repositories have been developed for archiving and disseminating $\mathrm{CH}$ data, thus improving the accessibility and visibility of digital $\mathrm{CH}$ resources. The MICHAEL project (MICHAEL Project Consortium, 2021) provides access to European digital collections from museums, archives, libraries and other cultural institutions. The ICOMOS Open Archive: Eprints on Cultural Heritage is a repository developed by the UNESCO-ICOMOS Documentation Centre (ICOMOS, 2021), containing the institutional archive of the scientific documentation produced by ICOMOS and documentation related to $\mathrm{CH}$ conservation produced by the international scientific community. In the same context, HeritageCare project has developed a web-GIS system for the management of HBIM models on top of a complete structured database (Mora et al, 2021). The cataloguing framework contains all the information needed for monitoring activities and maintenance actions (Sánchez-Aparicio et al., 2020).

In addition to $\mathrm{CH}$ repositories like the aforementioned ones, which do not permit any visualization of 3D content, several repositories of 3D CH models have been created in recent years. Patel et al. (2004) implemented a system for the digital capture of museum artefacts, which are accumulated into a multimedia repository comprising 3D models, images, video and sound clips as well as textual descriptions, within the ARCO project. The CyArk 3D Heritage Archive (CyArk \& Partners, 2020) is an effort that began in 2003 by the non-profit organization CyArk to create an online repository for 3D CH data, generated via laser scanning and image-based photogrammetric techniques. The 3D models may be viewed either by their online 3D viewer, or in the form of a video. Also, virtual and guided tours are supported by CyArk's site. Doerr et al. (2010) designed an integrated repository tested in the field of $\mathrm{CH}$, to ingest, store, manipulate and export complex digital objects, their components as well as related metadata and enable efficient access to the information, within the 3D-COFORM project. Felicetti and Lorenzini (2011) reported the development of a semantic enabled and persistent repository, where 3D CH models as well as any kind of digital data and metadata can be stored, retrieved and shared. Another project related to the development of a repository and platform for 3D CH models is MayaArch3D (Auer et al., 2014). Its main aim was the development of a web platform that stores spatial and non-spatial data, permitting visualization of 3D models and performance of GIS analyses on them.

Dhonju et al. (2018) developed an online geo-crowdsourcing system empowered by web-mapping for data visualization, termed Share Our Cultural Heritage $(\mathrm{SOCH})$, which may be utilized for large-scale $\mathrm{CH}$ documentation and sharing. The platform collects geo-located $\mathrm{CH}$ data from the public and supports, among others, web-based generation of 3D models from images and visualization of 3D $\mathrm{CH}$ models. Nishanbaev (2020) reported a new methodology to build a web repository for 3D CH models based on an open-source, database-driven content management system which integrates maps, 3D models, and geospatial data of $\mathrm{CH}$ objects. What is more, OMEKA is a wellknown platform for $\mathrm{CH}$ institutions that may work as a web repository, offering 3D storage and 3D visualization features (Corporation for Digital Scholarship et al., 2021). There are also some well-established commercial repositories available on the Internet (e.g., Sketchfab, TurboSquid) that may be used for storing, publishing and visualizing 3D CH models.

Furthermore, significant research, related to the work introduced in this paper, has been conducted on the fields of database development for management of different kinds of $\mathrm{CH}$ information (Kioussi et al., 2019), web information system development for management and dissemination of $\mathrm{CH}$ data (Meyer et al., 2007) and development of a prototype for the management and context-driven browsing of $\mathrm{CH}$ environments (Amato et al., 2017). Koller et al. (2009) reported a number of technical research challenges that should be addressed to develop an archive of 3D $\mathrm{CH}$ models, including, among others, management of rights for the 3D models and effective metadata structures, interoperability and 3D searching within an effective organizational structure of the archive. These challenges are addressed within the developed web-based platform. The work presented in the paper differs from previous works like the aforementioned ones, in a way that the proposed web-based platform supports not only visualization of 3D and 2D data of heterogeneous origin and format, retrieval of multimedia information and integration of tools for interaction with the 3D scene, but also differentiation of its content and interactive services based on the scientific specialty and the field of interest of its users. In this way, thanks to its personalized content, it serves the different requirements of different user categories.

\section{METHODOLOGICAL APPROACH}

The proposed methodology for the concept, design, and implementation of the various components of the platform is presented in this section. After discussing about the 4D visualization pipeline and its technological tools, an overview of the content personalization based on user's profile is described. Finally, insights into the back-end system comprising its specifications, the structure of the relational database and the administrator interface are provided.

\subsection{D Web Visualization - 3DHOP}

The 3D rendering and visualization of the high-resolution textured models is assigned to 3DHOP, an open-source framework developed by the Visual Computing Laboratory of ISTI - CNR (Potenziani et al., 2015). Loading a high-resolution model in its entirety requires the transfer of a file of hundreds of megabytes in the network. This technique is ineffective, especially if the visualization occurs after the completion of the transmission. In order to balance latency and avoid lossy data compression, the multiresolution approach of the Nexus library is employed (Ponchio and Dellepiane, 2015). The 3D models are converted into the .nxs format that enables progressive and view dependent loading. The difference in resolution (fourth dimension - 4D) is achieved by introducing the models as structures of different levels of detail; the structure of the proper level of detail is dynamically displayed as the user navigates within the 3D scene.

Among the plethora of 3D graphics libraries and frameworks, the 3DHOP platform excels in performance, supports Nexus's view dependent resolution and falls in the scope of $\mathrm{CH}$ field. As it has been designed to handle detailed and large-sized 3D representations, the potential of maintaining original fidelity and 
visual quality during visualization is high. It is based on the WebGL graphics API and the SpiderGL utility library, running inherently in every modern browser. For intuitive interaction with the 3D data and interoperability with HTML elements, it exploits the JavaScript programming language in a client-side architecture. Despite its imperative paradigm, the 3D scene adopts a declarative-style configuration and it is divided into the following logic blocks: meshes, instances, trackball and space. Finally, it is coupled with pre-built functionalities, such as a measuring suite, lighting controller, dynamic models sectioning etc. as well as exposed functions embracing further programming and expansions. As it is not accompanied by a management application, these functions are limited to the parameters and values that each programmer forms during the coding process. The pre-built tool of 3DHOP that is further extended in the current work is "Hotspots". Traditionally, clickable basic geometries are loaded on top of the surface of the main 3D model to indicate an area or point of interest. Any addition or configuration occurs with front-end programming and its result is visible only once the entire 3D scene is reloaded. The proposed expansion maps this process to a back-end management panel, previews the position and appearance of each hotspot and attaches multimedia to each of them in an automated and simplified way.

\subsection{Multi-user Approach}

Both the content and the interactive services of the METEORA web-based platform are differentiated, based on the scientific specialty and the field of interest of its users, serving their different needs and requirements, based on properly structured scenarios of use (Ioannidis et al., 2020). Thus, data of heterogeneous origin, format and field of interest are handled and correlated within the platform and different services are available to different kinds of users. As far as the content of the platform is concerned, spatial data (e.g., 3D models) and non-spatial data (e.g., textual information, images, videos) related to the historical, religious, cultural, architectural and geopolitical aspects of the two rocks of interest as well as the other monuments of the Holy Land of Meteora are integrated within the platform. The following categories of users have been defined: (i) tourists/simple (unauthorized) users; (ii) geospatial engineers; (iii) archaeologists/architects/conservators; (iv) historians/ philologists/theologians/priests; (v) educators; (vi) business entrepreneurs; and (vi) $\mathrm{CH}$ authorities. The access to the basic functionalities, tools and content of the METEORA platform is free for all users. In this general case, the category of tourists/simple users belongs. The other categories of users are provided with the possibility to create an account, so that, after logging in with their credentials, they can redirect to personalized content and access to services based on their specialty. The following services have been developed and are available to all users, without any authentification: (i) 4D multi-scale visualization system; (ii) interaction tools with the 3D scene; (iii) mechanisms for accessing the content of the platform and retrieving multimedia; and (iv) $2 \mathrm{D}$ map that supports visualization of 3D models superimposed on it. Additional services and tools are available to authorized users, as described in detail in section 4.3.

\subsection{Back-End System and Control Panel}

3.3.1 System Specifications and Database Schema: The proposed back-end system comprises a Relational Database Management System (RDBMS) and a relative administrator interface pivoted to the requirements of the 3D viewer, the interactive services of the platform and the heterogeneity of the content. For maintaining the multi-user approach and creating the 3DHOP's management environment, an analysis of the database ontology is needed. To attain maximum adaptability, the following specifications are defined:

- 3D models storage in the database (CRUD);

- mechanism for simultaneous 3D models display;

- point and metadata management (CRUD);

- relation mechanism between models and points;

- attributes management (CRUD);

- attributes attribution per point (CRUD);

- place registration and metadata management (CRUD);

- relation mechanism between places and points (CRUD);

- relation mechanism between points and multimedia (CRUD);

- search logic development and data return; and

- datasets returned per user's role and rights.

The tables and correlations of the database are designed in the PHPMyAdmin open software, based on the criteria of long-term sustainability and flexibility. Tables provide the method to develop data structures, correlations between them and queries with joins corresponding to specific data fields (Kanellakis, 1990). Executed queries are wrapped in functions that could include conditions, counters, variables and actions in order to configure any data access and search mechanism. In Computer Science, the fields of tables are related under three different types: "one to one" (1-1), "one to many" (1-*) and "many to many" (*-*) (Codd, 1989). Each relationship follows a specific programming approach, with the most difficult being formulated by "many to many". For the “*_*” relation, a conjunction table is required, where the id of each related table ends up as a foreign key in the table. The structure level of the relationship permits the performance of more or less complex queries using MySQL, which return more or less complex results.

3.3.2 Database Entity Design: Based on the specifications of the application, two main entities emerge for the database design: "Point" and "Person". The functionality for the "Point" entity focuses on spatial information and data management, while the functionality for the "Person" entity focuses on system configuration and access to the available data, depending on the user's role. The "Point" entity is the data container that defines the following 3DHOP's and DOM's (Document Object Model) elements: point; place and 3D model relations; point metadata definition (geographical information, title, description, attribute); point projection properties (model type, position, size, rotation, coloring); and point associations with multimedia files and other points. The "Person" entity handles the metadata per registered person (name, surname, mail, password); the role per person depending on the information fields and the functionality of the available tools; and the application's usage rights per role (curator and administrator with CRUD rights).

\subsubsection{Usability and Functional Principles for Back-End} Interface: The role of the administrator interface is twofold: it serves as a complete management environment for 3DHOP and a back-end control panel. It manages, automates and dynamically manipulates all the main functionalities of 3DHOP for non-expert administrators. It permits the management of 3D models, places, hotspots and their relative attributes as well as the geometries and the multimedia files that are attached to each hotspot. As a database control system, it enables the CRUD-based configuration of the back-end pages that support full administration rights. Thus, it has an easy-to use and responsive Graphical User Interface (GUI) that comprises various 3D and 2D viewports for real-time data preview, along with buttons, lists and other types of inputs for mapping the described operations. 

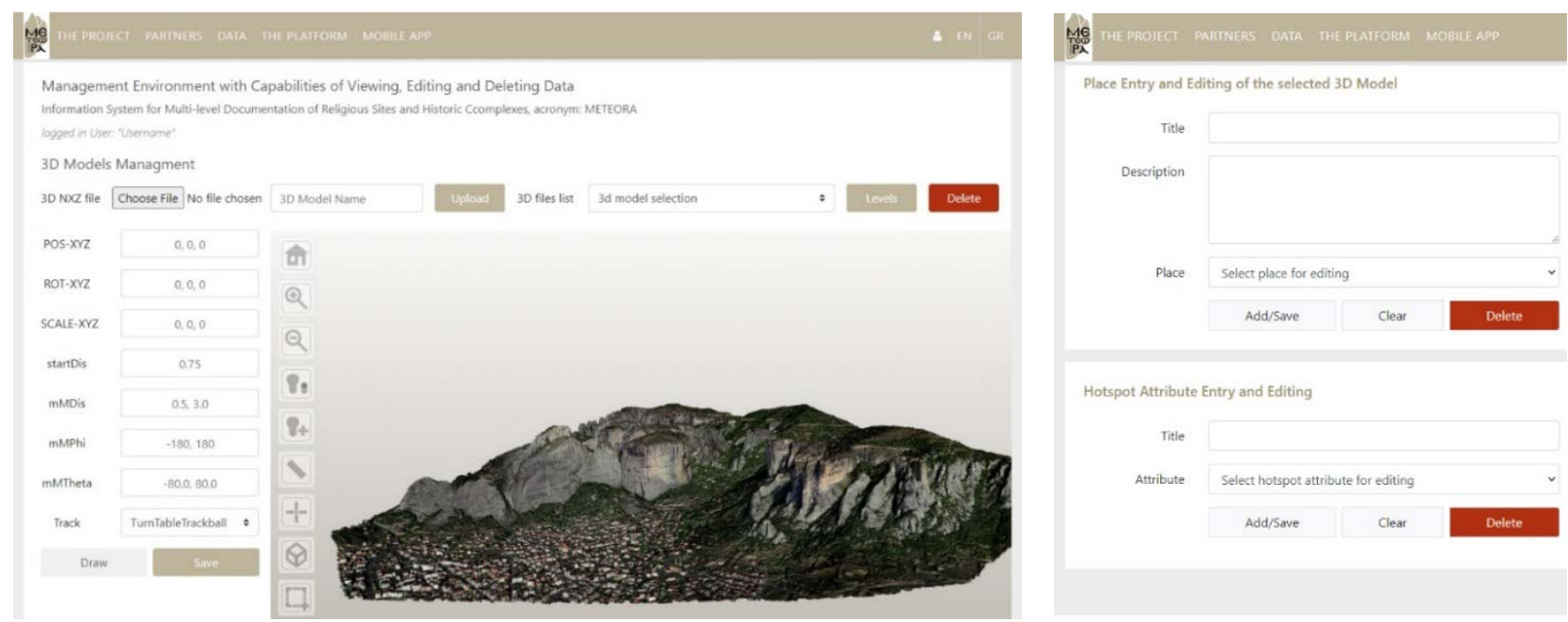

Figure 1. The management environment of METEORA platform. Left: 3D models loading, scene configuration and preview. Right: Definition of place and attribute values for each 3D model and hotspot.

To showcase the functionalities of the interface, a usability scenario is discussed. When the administrator logins and uploads a 3D file, the list of the available models for editing is updated (Figure 1, left). By selecting the desired 3D model, the options that determine its position, rotation and size in the 3D scene are activated for modification and preview. Further adjustable options are the parameters of the camera, namely, the start distance, the minimum and maximum transform range, the track type and the Phi and Theta angles. After defining the appearance of the selected 3D model, the admin has the ability to add, edit and delete places and hotspots on it. The editable fields for hotspots registry are the following: title; description; shape; color; transparency; position; rotation; and size on the model. Every configuration can also be displayed on the 3D viewer in real time. Hotspots can be linked with one or more attributes and with one or more places (Figure 1, right). The options on attributes management (CRUD controls) are provided on a second page. Attributes are properties that hotspots carry, in order to be grouped according to search criteria. The admin can link them to one or more hotspots by simply selecting them from a list. Examples of such attributes are "point of view", "walking route", "place of cultural interest”, "geophysical monument”, etc. Then, a third admin page determines the 3D shapes that are used to highlight the area or point of interest of the surface of the 3D model. They serve different interaction needs and can vary from simple to complex geometries. The administrator has the possibility to upload 3D models that could serve as relevant hotspots geometries (sphere, pyramid, polygon, trapezoid, etc.). Finally, the fourth management page concerns the multimedia management. Images and videos can be uploaded along with their title, description and alternative ("alt") text and can be linked to one or more hotspots.

\section{IMPLEMENTATION}

This section concerns the methods, paradigms and technological tools used for the development of the back-end and front-end systems of METEORA platform. After the presentation of the overall platform's architecture, emphasis is given on the database management system. The tables and operational characteristics of each entity as well as the generic database design logic are presented in detail. Moreover, an innovative data loss control mechanism is analyzed and illustrated. Finally, a concise description of the front-end tools and services for the various user's categories is provided.

\subsection{Platform Architecture}

The system architecture of the METEORA platform lies on the 3-Tier Architecture model, which distributes in an optimal way the individual components based on their functionality and applicability. In particular, it assigns to the Presentation Layer the implementation of the required web visualizations (3D Viewer, Asset Viewer), the search and retrieve service and the interaction tools (Search \& Retrieval, 2D Map). The Application Layer undertakes the client/server requests and responses control of the 3DHOP's management system (Figure 2, left). Finally, in the Persistence Layer, a database management system (DBMS) organizes, manages and provides access to the input data. The platform is implemented with the LAMP stack, i.e., based on the combined use of Linux, Apache server, MySQL and PHP. Its technologies are open source with transparency and absolute compatibility with each other. At the Presentation Layer, the visualization system is based on the 3DHOP framework and the Three.js graphics library. Any service or tool that is directly related to the 3D space will be developed by extending or modifying the existing codebase. The JavaScript library jQuery and Bootstrap toolkit are also integrated for events handling and responsiveness respectively. At the Application Layer, a RESTful service runs on the Apache web server and is developed by the PHP scripting language. Additional frameworks are used to control access and content management based on users' roles. Finally, the MySQL DBMS is responsible for the storage and indexing of all the platform's structured data on top of the Apache server in a Linux environment (Figure 2, right).

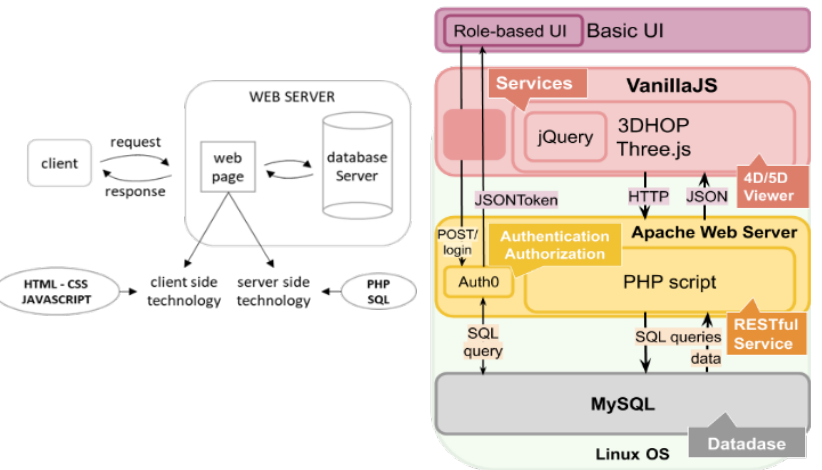

Figure 2. System architecture of METEORA platform. Left: Client-side to server-side communication. Right: Technological tools and interactions of the 3-Tier Architecture model. 


\subsection{Database Management System}

The back-end management system aims at the design, implementation and optimization of all the entities, relationships and functions of the database, in terms of spatial dimension and multi-user approach. In order to ensure smooth information flow and data input in the 3DHOP viewer as well as to formulate the logic that conceptually develops the application, the database scheme of Figure 2 has been created.

4.2.1 The "Point" Entity: In the following, the database tables for the "Point" entity are described, as they are illustrated in Figure 3. The "Model" table carries information for the 3D models (i.e., position, rotation and size of model, camera movement types, model name, route path) in conformance with the specifications of the 3DHOP viewer. The "Hotspot" and "HS Model Type" tables are also 3DHOP data containers in which information about the 3D display of hotspots lie (i.e., location, size, model). The "Hotspot Attribute" and "Place" tables are required for the conceptual configuration of the application. The labels for each hotspot are formed by their attributes (e.g., cultural route, sport activity), allowing to thematically develop the application context. Respectively, the "Place" table constitutes the tool for grouping hotspots in spatial sections. The "Hotspot Attribute", "Place", "Image" and "Video" tables have a "many to many (*-*)" relation with the "Hotspot" table, i.e., an attribute/place/image/video can be related with many hotspots and a hotspot could be related with many attributes/places/images/videos. To develop the “*_*” relationship, conjunction tables are needed (i.e., "HS-Hotspot Attr”, "HS-Place”, "HS-Image” and "HS-Video”, respectively). Metadata tables ("Hotspot Metadata", "Attribute Metadata", "Place Metadata", "Image Metadata" and "Video Metadata") have been created to support the second language ("Language" table). The way they are organized aims at the flexibility and scalability of the system, as it allows the integration of more languages with the maximum functional and administrative adequacy. In conventional and simplified programming, the language field is integrated in each respective table. The proposed scheme entails the creation of the Metadata table and more complex relationships but achieves the multiplication of the records in each table and, accordingly, a quick and effortless update when a new language should be supported.

4.2.2 The "Person" Entity: The METEORA platform registration is divided into the following categories: system administrators; users who register for study and data retrieval purposes; and users who develop a related business activity in the case study area. To meet the needs of different rights per user, the design described in the following, concerning the "Person" entity, has been adopted, as illustrated in Figure 2. As explained in the previous section, the metadata tables are used for structuring data into independent tables, to support multilingualism. The "Person" table, which refers to those ones who may register in the application, is related to the "Person Metadata", "Role", "Corporate" and "User" tables. The "Role" table is used to give a role to each person. "Role" properties enable the determination of the permission levels per user (viewer tools, data retrieval). Each person may be assigned only one role. The "User" table determines if the user has admin rights; each user corresponds to one person and has a specific management level in the application. The "Corporate" table serves the needs for the registration of companies operating in Kalampaka, Kastraki and the wider area of Meteora, in the platform. It has relations with the tables "Corporate Metadata”, "Person", "Corporate Type" and "HS-Corporate". The relation "Corporate" - "Person" provides the link between person and company and the relation "Corporate" - "Corporate Type" provides the link between company and company type or types. Between the "Corporate" and "Hotspot" tables, a "*_*” relation is defined, using the conjunction table "HS-Corporate". In this table, there is a double “*_*” relation, which is a relatively rare version of relationship, as it increases the programming difficulty during "insert" and "update" processes. However, its main advantage is the fact that it returns data using one less "SELECT" query in the database, as explained in section 4.2.3. Therefore, this dual relation simplifies the formulation of complex SQL queries in the database.

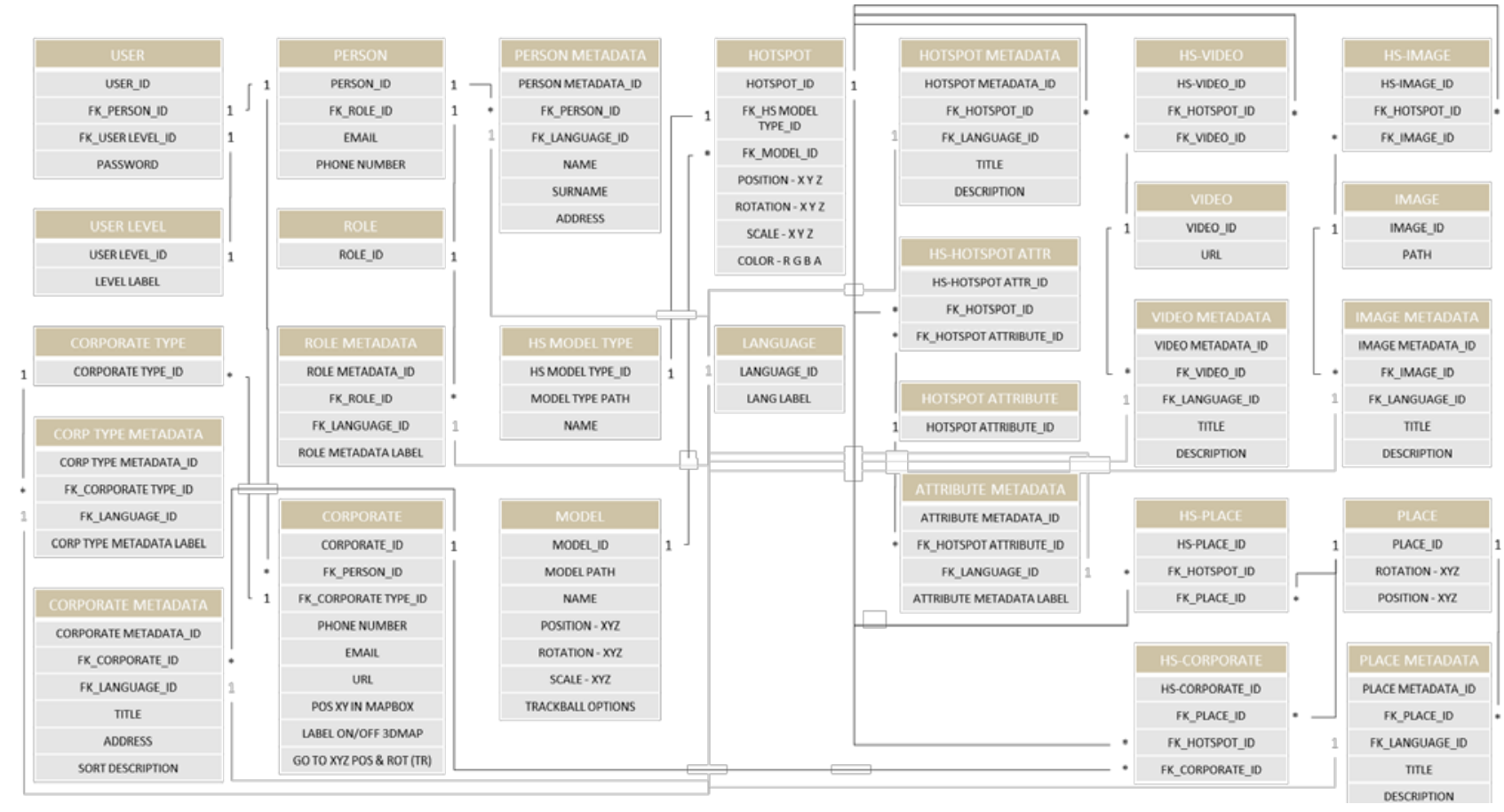

Figure 3. Database relational entity diagram where the structure of tables and the properties of correlations are based on the interactive services of the platform. 
4.2.3 Database Model Efficiency: The METEORA platform database consists of 28 tables and a significantly larger number of associations. While the Database Model Entity Diagram seems rather complicated, it is designed under the criteria of system sustainability, normalisation and flexibility. Each relationship between tables is useful, as it can be clarified in the paradigm that follows. The "Place" table is related with the "Hotspot" table through the "HS-Place" table. Respectively, "Corporate" is related with "Hotspot" through "HS-Corporate". To define the correlation between "Corporate" and "Place", a double "*_*" relation is defined in the "HS-Corporate" table. In the case that the "HS-Corporate" table creation was omitted, an additional "SELECT" query in the database would be required to get the relation between "Corporate", "Place" and "Hotspot", as illustrated using the following case study scenario: "I live in the 'hotel name' and I want to know which hotspots are around it". The corresponding SQL queries are illustrated in Figure 4.

- SQL query 1: No use of the "HS-Corporate" table. To get the relation between "Corporate" and "Hotspot", the corporate id is added as a foreign key to the "Place" table. The corresponding SQL query may be explained as follows. The company id is found using the company name (4th "SELECT"); the related place is found using the company id (3rd "SELECT"); the relevant hotspots are found based on the place returned, (2nd and 1st "SELECT"); from the 1st "SELECT", the related hotspots names are returned. The 2nd "SELECT" is performed with INNER JOIN, as the "Place" table is associated with "Hotspot" through "HS-Place".

- SQL query 2: Use of the "HS-Corporate" table. As in the previous case, the corporate id is found using the company name (3rd "SELECT"); from the "HS-Corporate" table, all hotspots associated with this corporate id (2nd "SELECT") are directly returned; finally, the names of the associated hotspots are returned (1st “SELECT”).

In the case where the "HS-Corporate" table was omitted, by registering 100 points in the database, at least 400 "SELECT" actions would have to be executed;

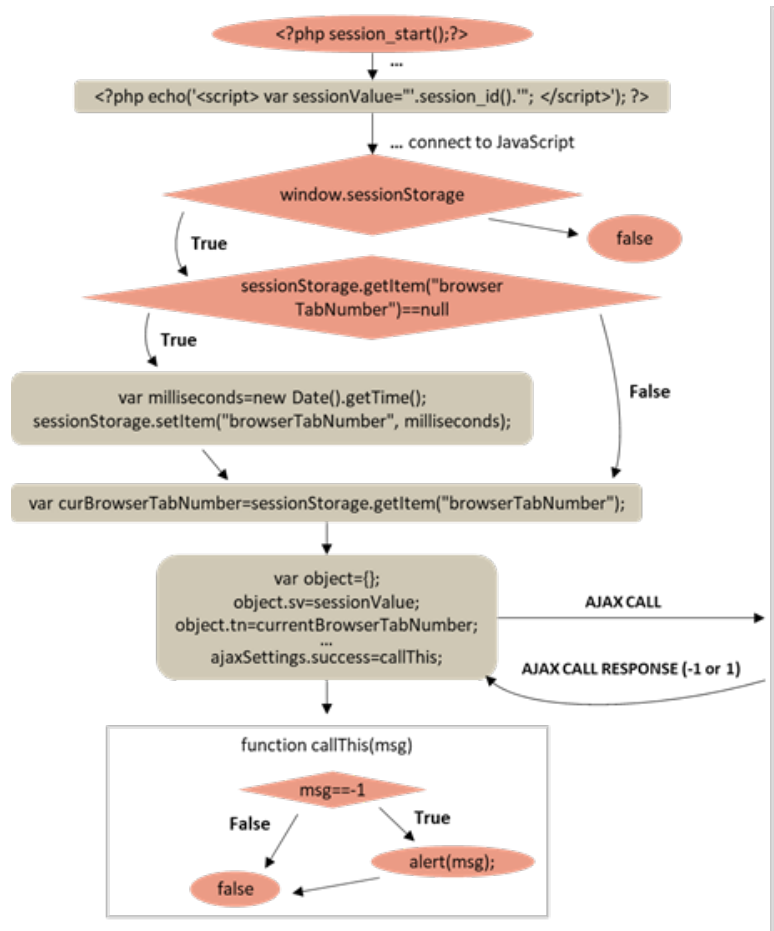

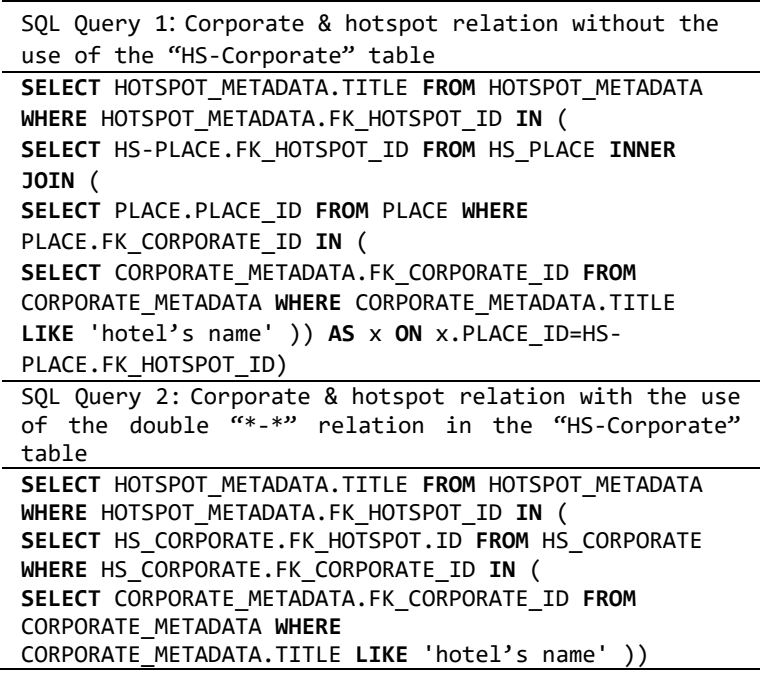

Figure 4. SQL queries for finding hotspots near a hotel developed with two different methods.

in the developed SQL query, 300 "SELECT" actions have to be performed. The number of queries depends on the numbers of points and user searches. In "small" systems, it does not play a significant role. However, in larger ones, it contributes significantly to the application response time.

4.2.4 Data Loss Control Mechanism: Data loss may occur in submission forms during the "update" process. The reasons may be different, e.g., simultaneous CRUD operations at the same record by two logged in users; multiple instances of the application running simultaneously in different browser tabs; lost connection of database; design error during the development process. An administrator may open the application in several browser tabs to manage multiple records simultaneously. If any tab (1st tab) carries the same record id with another tab (2nd tab), then during the "update" process, only the data from the last submitted form will be saved (i.e., the 2 nd one). In fact, if the

Figure 5. PHP \& JavaScript source code logic for terminating a 2nd instance of the application at the same browser. 
user returns to the 1 st tab and continues editing, during the "update" process the saved data from the previous action will be deleted: only the data of the 1st tab will be saved. Although the scenario is caused by user's mishandling, as the operating behavior is correct, a data loss control mechanism is required. For this purpose, a control mechanism that does not allow the user to open the same record id in different tabs is proposed and developed. The mechanics for the function are described as follows. As a session starts, client-side JavaScript variables are saved to server-side PHP variables (session, record-id and browser tab name). Session association with record id and browser tab name is created and saved in the database. As another session starts, it is checked through the association, if it concerns the same record id and either an approval or a close action is executed. By closing the browser's tab, the function which deletes the association from the database is called, so the application continues to run smoothly. The same function is performed when the PHP garbage collection mechanism is triggered (e.g., if the user remains inactive for a certain period of time, the session between client and server is terminated). The diagram of Figure 5 illustrates the source code to develop the basic part of the mechanism mentioned above. Here, session's id and browser's tab association are checked and validated through parsing. Then, the second application instance is terminated per session and not per record id, as it normally happens. The script is developed in two parts involving both server-side and clientside technological components. The client-side part uses JavaScript programming language and AJAX asynchronous calls while the server side lies on PHP scripting and MySQL.

\subsection{Front-End Tools and Services}

The creation of the front-end interface of the METEORA platform involves the development of the $4 \mathrm{D}$ viewer as well as the tools of interaction and data retrieval and the design of the graphical user interface. The Three.js graphics library and the 3DHOP framework have been used for the development of the viewer; they support visualization of high-resolution 3D models along with interactive navigation, data recovery from the backend interface and spatial interconnection of the information that defines and clarifies the 3D scene. Within the 4D visualization system, interaction tools with the $3 \mathrm{D}$ scene have been implemented (i.e., measurement tools, tool for changing the scene lighting, tool for highlighting areas of interest), in addition to standard navigation method (panning, zooming and rotating). According to its $4 \mathrm{D}$ visualization system, the resolution of the models is constantly being optimized, depending on the distance of the navigation camera. Moreover, mechanisms for accessing the content of the platform and retrieving multimedia and other kinds of information have been developed. The retrieved information and multimedia work in coordination and in combination with the 3D models for achieving direct spatial correlation. Furthermore, a 2D map that additionally supports visualization of 3D models is part of the METEORA platform.

The multi-user approach described in section 3.2 was implemented by providing specific personalized tools and services to authorized users of the platform, depending on their specialty and field of interest. Unauthorized users, i.e., simple users who visit and use the platform without creating an account, fall into the category of tourists. These unauthorized users have the possibility to navigate in a textured 3D model of the site of interest, either by choosing among several available alternative routes of "supervised" navigation, or through free navigation. The "supervised" navigation in various parts of the scene is accomplished automatically through seamless movements of the 3D model and the camera; successive transitions within the 3D space along with rotations and changes in the viewing angle provide them with a complete understanding of the scene. What is more, information is automatically displayed about the projected landmark in a text field above the 3D scene. The synchronization of the display and the corresponding information provides a clear and critical overview of individual points of interest. Alternatively, they may perform a free manual navigation with the possibilities to rotate, pan, zoom in/out and interact with points on the map and in the navigation menu. Additionally, theses unauthorized users may see dynamic information for user-specified points of interest during navigation in a 3D model. Apart from 3D navigation possibilities, they may navigate in a 2D map of the area of interest, which additionally supports visualization of 3D models superimposed on the map. Also, they have the possibility to search, through the platform, for information about the site of interest. Finally, a tool that allows them to perform a localized virtual tour during their visit in a site of interest, provided that they have a mobile device with enabled GPS sensor is also available via the platform. In the following, the additional tools and content provided to the authorized users of the platform are described, based on their specialty.

Geospatial engineers have access to (i) measurement tools of coordinates and distances, that may be applied on the surfaces of the 3D models; (ii) the metadata of 3D models; (iii) collections of aerial images and architectural plans; (iv) collection of additional 3D models for visualization and interaction within the platform; and (v) a form in order to contact the administration for permission to provide them with the requested geospatial data. Archaeologists/architects/ conservators have access to (i) measurement tools of coordinates and distances; (ii) collections of aerial images and architectural plans; (iii) collection of additional 3D models for visualization and interaction within the platform; and (iv) specialized content (text and images) for the 3D reconstruction of the Monastery of St. Modestos. Historians/Philologists/Theologians/Priests have access to (i) additional text, image and video content about the monuments of the Holy Land of Meteora; (ii) searching and retrieval of specialized information using the provided search field; (iii) collections of aerial images and architectural plans; and (iv) collection of additional 3D models for visualization and interaction within the platform. Teachers have access to (i) additional text, image and video content about the monuments of the Holy Land of Meteora; (ii) searching and retrieval of specialized information using the provided search field; and (iii) collections of images and videos. Entrepreneurs have access to a contact form in order to integrate to the platform their local businesses, in the fields of tourism and commerce, which operate in towns and villages near (Kalampaka, Kastraki) or in the greater area of Meteora. Finally, CH Authorities have access to a contact form for the submission or request of $\mathrm{CH}$ data.

\section{CONCLUSIONS}

This paper presents the development of a multi-user online platform for the management, visualization and dissemination of 3D and 2D CH datasets. The platform consists of three building blocks: a RDBMS, an administration panel with a user-friendly interface and a front-end 3D visualization system based on 3DHOP. The work conducted in this paper emphasizes on the mid-level software that abstracts the underlying back-end operations to a complete 3DHOP web management environment. The proposed system has increasing efficiency in authoring, creating, managing and uploading 3D models along with their relative multimedia directly in the $3 \mathrm{D}$ scene of a $3 \mathrm{DHOP}$ viewer. A flexible database scheme supporting multiple users and easy 
integration of different languages as well as innovative data loss methods have been developed to enhance the back-end infrastructure. In the front-end interface of the platform, 3D models of multi-resolution format along with metric and nonmetric information are visualized and correlated in a clear and educational way, through interactive access and retrieval services. Thus, data of heterogeneous origin, format and field of interest are easily handled by non-expert administrators and disseminated to different user categories. The platform and its individual subsystems can be employed as standards for documenting sites of archaeological, historical or religious significance.

\section{ACKNOWLEDGEMENTS}

This research has been co-financed by the European Union and Greek national funds through the Operational Program Competiveness, Entrepreneurship and Innovation, under the call RESEARCH-CREATE-INNOVATE (project code: T1EAK02859).

\section{REFERENCES}

Amato, F., Moscato, V., Picariello, A., Colace, F., Santo, M. D., Schreiber, F. A., Tanca, L., 2017. Big Data Meets Digital Cultural Heritage: Design and Implementation of SCRABS, A Smart Context-awaRe Browsing Assistant for Cultural EnvironmentS. Journal on Computing and Cultural Heritage, 10(1), 1-23.

Auer, M., Agugiaro, G., Billen, N., Loos, L., Zipf, A., 2014. Webbased Visualization and Query of semantically segmented multiresolution 3D Models in the Field of Cultural Heritage, ISPRS Ann. Photogramm. Remote Sens. Spatial Inf. Sci., II-5, 3339.

Codd, E. F., 1989. Relational Database: A Practical Foundation for Productivity. In Readings in Artificial Intelligence and Databases, pp. 60-68, Elsevier.

Corporation for Digital Scholarship, Roy Rosenzweig Center for History and New Media, George Mason University. 2021. OMEKA. https://omeka.org/ (7 January 2021).

Champion, E., \& Rahaman, H., 2020. Survey of 3D digital heritage repositories and platforms. Virtual Archaeology Review, 11(23), 1.

CyArk \& Partners, 2020. CyArk Collections. https://www.cyark.org/ (7 January 2021).

Dhonju, H. K., Xiao, W., Mills, J. P., Sarhosis, V., 2018. Share Our Cultural Heritage (SOCH): Worldwide 3D Heritage Reconstruction and Visualization via Web and Mobile GIS. ISPRS Int. J. Geo-Inf., 7, 360.

Doerr, M., Tzompanaki, K., Theodoridou, M., Georgis, C., Axaridou, A., Havemann, S., 2010. A Repository for 3D Model Production and Interpretation in Culture and Beyond. 11th Int. Symposium on Virtual Reality, Archaeology and Cultural Heritage, 97-104.

Felicetti, A., Lorenzini, M., 2011. Metadata and tools for integration and preservation of cultural heritage 3D information. Geoinformatics FCE CTU, 6, 118-124.

ICOMOS, 2021. ICOMOS Open Archive: Eprints on Cultural Heritage. http://openarchive.icomos.org/ (7 January 2021).
Ioannidis, C., Verykokou, S., Soile, S., Boutsi, A.-M., 2020. A multi-purpose cultural heritage data platform for 4D visualization and interactive information services, Int. Arch. Photogramm. Remote Sens. Spatial Inf. Sci., XLIII-B4-2020, 583-590.

Kanellakis, P. C., 1990. Elements of Relational Database Theory. In Formal Models and Semantics, pp. 1073-1156, Elsevier.

Kioussi A., Karoglou, M., Doulamis, A., Fragkoudakis, C., Potikas, P., Protopapadakis, E., Delegou, E., Alexakis, E., Moropoulou, A., 2019. A Knowledge Json-Based Database for Integrating Multiple Disciplines in Cultural Heritage. Nondestructive Evaluation and Monitoring Technologies, Documentation, Diagnosis and Preservation of Cultural Heritage, 121-132.

Koller, D., Frischer, B., Humphreys, G., 2009. Research challenges for digital archives of 3D cultural heritage models. ACM Journal on Computing and Cultural Heritage, 2(3), 17 p.

Meyer, É., Grussenmeyer, P., Perrin, J. P., Durand, A., Drap, P., 2007. A web information system for the management and the dissemination of Cultural Heritage data. Journal of Cultural Heritage, 8(4), 396-411.

MICHAEL Project Consortium, 2021. Multilingual Inventory of Cultural Heritage in Europe. http://www.michael-culture.org (7 January 2021).

Mora, R., Sánchez-Aparicio, L. J., Maté-González, M. Á., García-Álvarez, J., Sánchez-Aparicio, M., González-Aguilera, D., 2021. An historical building information modelling approach for the preventive conservation of historical constructions: Application to the Historical Library of Salamanca. Automation in Construction, 121(103449).

Nishanbaev, I., 2020. A web repository for geo-located 3D digital cultural heritage models. Digital Applications in Archaeology and Cultural Heritage, 16, e00139.

Patel, M., Walczak, K., Giorgini, F., \& White, M., 2004. A Cultural Heritage Repository as Source for Learning Materials. 5th Int. Symposium on Virtual Reality, Archaeology \& Cultural Heritage, 213-222.

Ponchio, F., \& Dellepiane, M. 2015. Fast decompression for webbased view-dependent 3D rendering. Proceedings of the 20th International Conference on 3D Web Technology - Web3D '15, 199-207.

Potenziani, M., Callieri, M., Dellepiane, M., Corsini, M., Ponchio, F., \& Scopigno, R., 2015. 3DHOP: 3D Heritage Online Presenter. Computers \& Graphics, 52, 129-141.

Pujol, L., Lorente, A., 2014. The Virtual Museum: A Quest for the Standard Definition. Archaeology in the Digital Era, pp. 4048. Amsterdam University Press.

Sánchez-Aparicio, L. J., Masciotta, M.-G., García-Alvarez, J., Ramos, L. F., Oliveira, D. V., Martín-Jiménez, J. A., GonzálezAguilera, D., Monteiro, P., 2020. Web-GIS approach to preventive conservation of heritage buildings. Automation in Construction, 118(103304).

Statham, N., 2019. Scientific rigour of online platforms for 3D visualization of heritage. Virtual Archaeology Review, 10(20), 1. 